\title{
A molecular dynamics simulation of polymer crystallization from oriented amorphous state
}

\author{
Akira Koyama $^{1}$, Takashi Yamamoto ${ }^{2}$, Koji Fukao $^{3}$, and Yoshihisa Miyamoto ${ }^{4}$ \\ ${ }^{1}$ Graduate School of Human and Environmental Studies, Kyoto University, Kyoto 606-8501 Japan, \\ ${ }^{2}$ Faculty of Science, Yamaguchi University, Yamaguchi 753-8512 Japan, \\ ${ }^{3}$ Department of Polymer Science and Engineering, \\ Kyoto Institute of Technology, Matsugasaki, Kyoto 606-8585 Japan, \\ ${ }^{4}$ Faculty of Integrated Human Studies, Kyoto University, Kyoto 606-8501 Japan
}

(Dated: October 31, 2018)

\begin{abstract}
Molecular process of crystallization from an oriented amorphous state was reproduced by molecular dynamics simulation for a realistic polyethylene model. Initial oriented amorphous state was obtained by uniaxial drawing an isotropic glassy state at $100 \mathrm{~K}$. By the temperature jump from 100 $\mathrm{K}$ to $330 \mathrm{~K}$, there occurred the crystallization into the fiber structure, during the process of which we observed the developments of various order parameters. The real space image and its Fourier transform revealed that a hexagonally ordered domain was initially formed, and then highly ordered crystalline state with stacked lamellae developed after further adjustment of the relative heights of the chains along their axes.

PACS numbers: 61.50.-f, 61.41.+e, 83.10.Rs
\end{abstract}

The molecular dynamics (MD) simulation has recently come to be recognized as a very promising tool to understand crystallization in systems of chain molecules, since they can directly provide us with molecular trajectories that are experimentally hard to access. In an early paper Rigby and Roe successfully reproduced a highly ordered lamella structure from a bulk amorphous state of $n$-decane [1]. The work was followed by studies that elaborated on detailed crystallization process in bulk $n$ alkanes of various length [2, 3]. Crystallization in thin films of $n$-alkanes was also investigated using MD technique [4, 5. Recent advances have come to enable the quantitative estimation of the growth rate vs. crystallization temperature [6].

Chain folded crystallization of polymers has also been investigated by molecular simulations. Kavassalis and Sundararajan first reported the MD studies on the collapse and folding of a single polyethylene molecule in vacuum [7, while Muthukumar and coworkers investigated a primary nucleation and subsequent crystal growth in solution by Langevin dynamics simulation [8, 9]. The molecular process of secondary nucleation and growth of lamella crystals has also been a subject of great interest and several investigations have been reported [8, 10, 11. We can notice in very recent literatures a surge of interest in reproducing chain folded crystallization from the melt and solution both during the primary nucleation [9, 12] and the growth [13], though at present we can only treat relatively short polymer chains of about hundreds of segments.

Crystallization of much longer chains in the bulk system is expected to require extraordinary long simulation time even by use of present day supercomputers, especially when we adopt realistic molecular models. We have already reported an MD simulation of polymer ordering in the bulk polyethylene [14]. We could there observe only the local ordering in chain packing, and we discussed the results within the context of recent experimental and theoretical investigations on precursor phenomena prior to crystallization [15, 16, 17, 18].

The polymeric material needs very long time until the onset of crystallization and can be easily quenched into amorphous glassy state with isotropic chain orientation. By drawing the amorphous sample below the glass transition temperature, we obtain the oriented amorphous state in which the chains run parallel to the draw direction on the average. We can then expect to accelerate the crystallization if we use such oriented amorphous sample, instead of isotropic melt [14, as an initial state of MD simulation. In fact, recent experiments revealed that the crystallization rates of polymers depend strongly on the degree of the chain orientation [19]. Moreover, it will be found that the oriented amorphous state is very convenient in investigating the spontaneous development of fiber structures with stacked lamellae or the detailed molecular level structure of the amorphous phase between lamellae [20].

In this Letter we report results of our recent simulation of polymer crystallization from bulk oriented amorphous state. The molecular model we adopted was essentially the same as that of our previous work [14]. We considered the energies of bond stretching, bond angle bending, and torsion of the united atom model of polyethylene (PE). The truncated Lennard-Jones 12-6 potential was used to reproduce van der Waals interactions between the atoms, where a rather short cutoff radius of $0.85 \mathrm{~nm}$ was used to improve computational efficiency. Bulk polymer system was made with a single linear PE composed of 5000 united atoms being placed in the MD cell under periodic boundary condition. Newton's equations of motion were integrated by the leap-flog algorithm with a time step of 4 $\mathrm{fs}$, and the temperature was controlled every 10 steps by the ad-hoc velocity rescaling, while the pressure was adjusted every 100 steps by the loose-coupling method [21. 


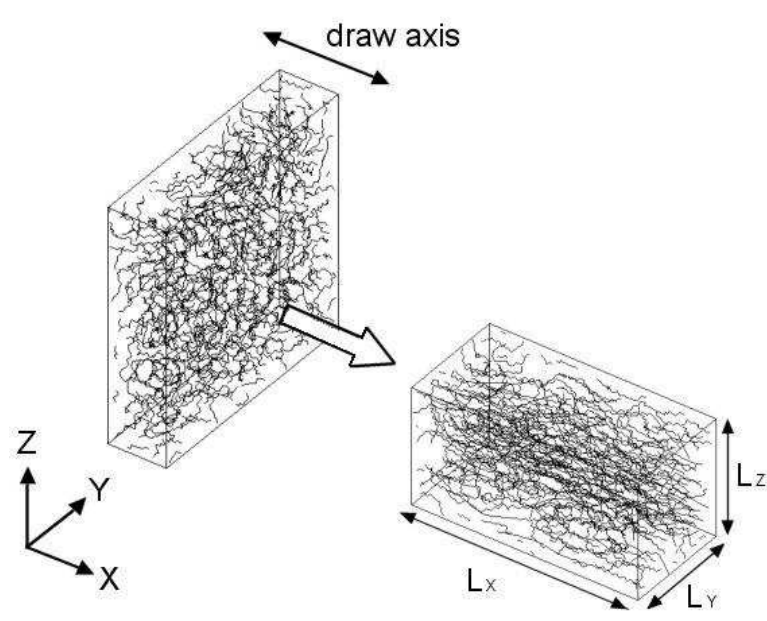

FIG. 1: Schematic picture of the preparation of oriented amorphous sample.

The parameter $\mathrm{M}$ used in the pressure control was set to be $13.26 \mathrm{~Pa} \cdot \mathrm{s} / \mathrm{m}$, and the shape of the MD cell was kept orthorhombic.

First we prepared the isotropic melt as follows. An initial configuration of the chain, with fixed bond lengths and bond angles, was generated using the technique developed by Theodorou and Suter [22], and then a short MD simulation of 10 ps under constant volume and temperature $(600 \mathrm{~K})$ was made to reduce the overlap of atoms. This initial state was then fully relaxed for $1 \mathrm{~ns}$ at constant temperature $(600 \mathrm{~K})$ and pressure $(1 \mathrm{~atm})$.

Next we generated the oriented amorphous state by drawing the isotropic amorphous sample well below the glass transition temperature. The glassy amorphous sample was drawn at $100 \mathrm{~K}$ along the $\mathrm{x}$-axis at a constant rate of $500 \% / \mathrm{ns}$ up to a final draw ratio of $400 \%$ (Fig.1), where the method of uniaxial drawing was the same as that used by Ogura and Yamamoto [23]. During drawing, the temperature was kept constant at $100 \mathrm{~K}$ and the lateral widths of the cell were allowed to shrink along the directions perpendicular to the draw axis under constant pressure $(1 \mathrm{~atm})$. After attaining the final draw ratio of $400 \%$, further relaxation was carried out for $1.5 \mathrm{~ns}$ under constant pressure (1 atm) and temperature $(100 \mathrm{~K})$.

Crystallization of the oriented amorphous state was then simulated for $30 \mathrm{~ns}$, under constant temperature $(330 \mathrm{~K})$ and pressure $(1 \mathrm{~atm})$. Figure 2 shows the changes with time of various quantities of the system. The internal energy slowly decreases until 10 ns (Fig. 2 (a)), but shows the steep reduction around 12 ns suggesting an onset of ordering. The specific volume also decreases almost lineally up to around 14 ns (Fig. 2 (b)), where the MD-cell shows clear initial shrink within 4 ns and then expands along the draw axis (Fig. 2 (c)) at the most pronounced rate around $12 \mathrm{~ns}$. Around $15 \mathrm{~ns}$, the MD-cell dimensions nearly come to saturate. It might seem strange that the specific volume increases again af- ter $15 \mathrm{~ns}$, but later this will be found an artifact due to the imposed periodic boundary condition and very slow relaxation in the well developed ordered state.

PE molecule has the lowest energy conformation trans and the second lowest gauche ${ }^{ \pm}$. Since the molecule has all trans conformation in the crystal, we evaluated the chain ordering by use of the trans fraction and the mean length of the trans sequence; the trans and the gauche bonds are here defined as the bonds whose torsion angles distribute around $0^{\circ}$ and $\pm 120^{\circ}$, respectively, within the range of $60^{\circ}$, whereas the length of the trans sequence is expressed by the number of the trans bonds in the sequence. The transition rates in Fig. 2(d) slowly decrease until around $10 \mathrm{~ns}$, but then they abruptly fall around 12 ns until the transitions nearly halt around $17 \mathrm{ns.} \mathrm{Both}$ the trans fraction and the mean length of the trans sequences in Figs. 2 (e) and 2 (f) increase steadily and show a marked jump up around 12 ns until they saturate completely around $17 \mathrm{~ns}$. The final state obtained after about $20 \mathrm{~ns}$ is thus considered to be made predominantly of the trans segments and highly ordered with very infrequent internal rotations between the trans and gauche.

In order to investigate the development of orientational order of the molecule, we examined the orientation of the C-C-C segments and the correlation volume that is defined as a measure of parallel alignment of the segments [1]. The orientations of the C-C-C segments along the three axes are evaluated by the mean squared cosines $<\cos ^{2} \theta_{X}>,<\cos ^{2} \theta_{Y}>$, and $<\cos ^{2} \theta_{Z}>$, where $\theta s$ are the angles between the orientation vector spanning every other carbons of the C-C-C segment and the axes. The correlation volume is defined as

$$
<V_{c}>=<\int_{0}^{r_{\max }} 4 \pi r^{2} g(r) s(r) d r>,
$$

where $g(r)$ is the radial distribution function of the center of mass for the segment, and $s(r)$ is the orientation correlation function between two segments defined as $s(r)=1 / 2\left(3<\cos ^{2} \theta_{i, j}(r)>_{i, j}\right)$, where $\theta_{i, j}(r)$ is the angle between the $i$-th and the $j$-th orientation vectors separated by $r$, and the average is taken over all pairs within the shell $r \sim r+\Delta r$, and the upper limit of the integration $r_{\max }=1.6 \mathrm{~nm}$. The chain orientation along the draw axis and the correlation volume were initially unstable, but started to increase around 4 ns until they saturate around $20 \mathrm{~ns}$ (Figs. 2(g), 2(h)). The rapid increase in the correlation volume around $12 \mathrm{~ns}$ indicates the spontaneous orientation and the parallel alignment of the segments.

The structural changes described above were analyzed by use of real-space image and its Fourier transform, the structure function. Three dimensional structure function is defined by

$$
S(\mathbf{q})=\frac{1}{N} \iint d \mathbf{r} d \mathbf{r}^{\prime}<\rho(\mathbf{r}) \rho\left(\mathbf{r}^{\prime}\right)>\exp \left(-i \mathbf{q} \cdot\left(\mathbf{r}-\mathbf{r}^{\prime}\right)\right),
$$

where $\mathbf{q}$ is the wave vector, $\mathbf{r}$ and $\mathbf{r}^{\prime}$ are the position vectors, $N$ is the total number of atoms. The atomic 

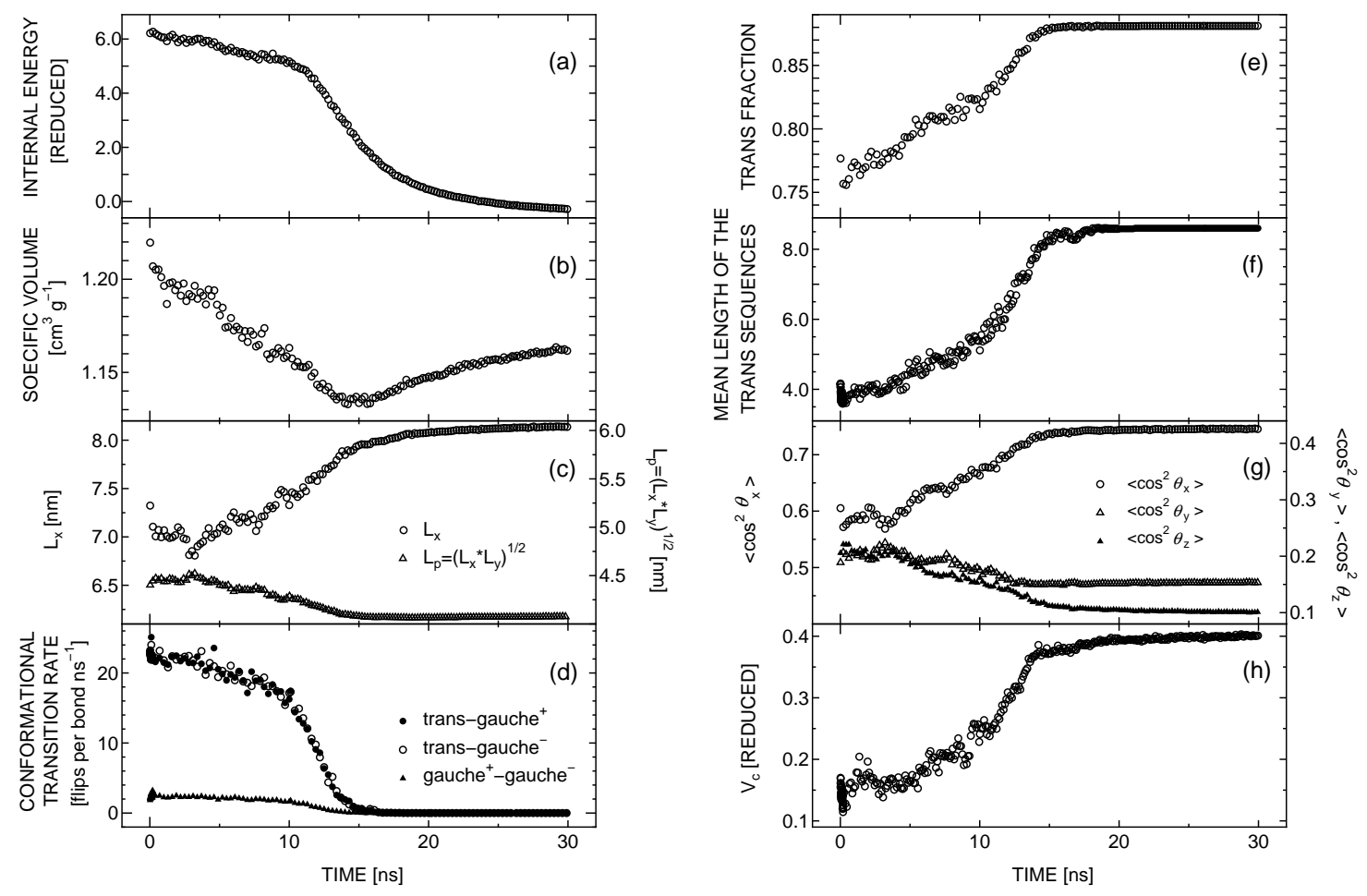

FIG. 2: Changes in the various quantities with time: (a) the internal energy, (b) the specific volume, (c) the side length of the MD cell, (d) the conformational transition rate, (e) the trans fraction, (f) the mean length of the trans sequences, (g) the degree of the orientation, (h) the correlation volume.

(a) $10 \underline{p s}$

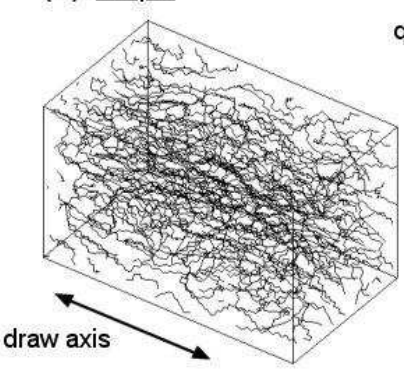

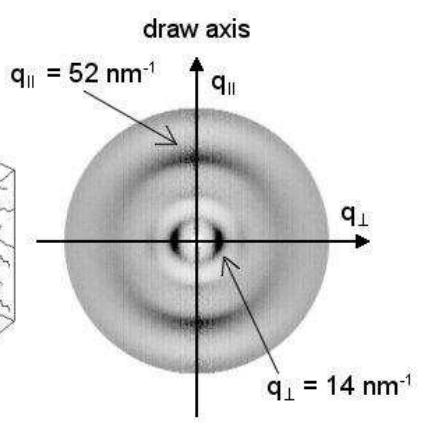

(b) $\underline{4}$ ns

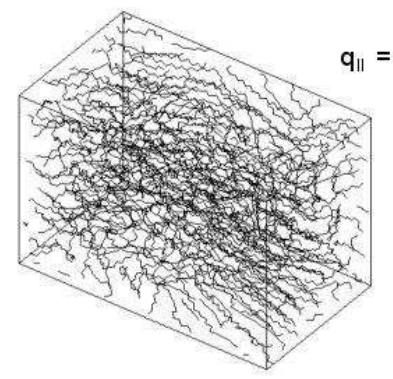

(c) $\underline{8 \mathrm{~ns}}$
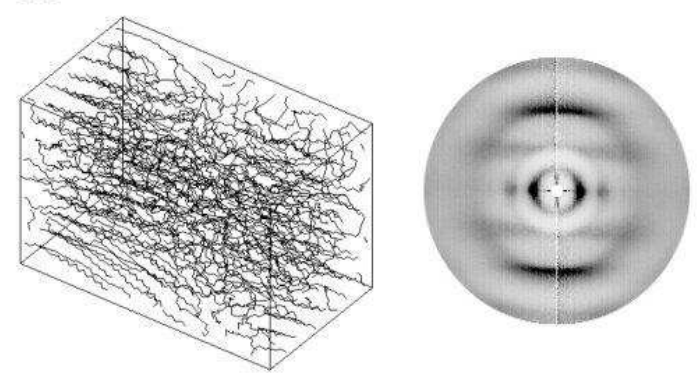

(d) $\underline{30 \mathrm{~ns}}$

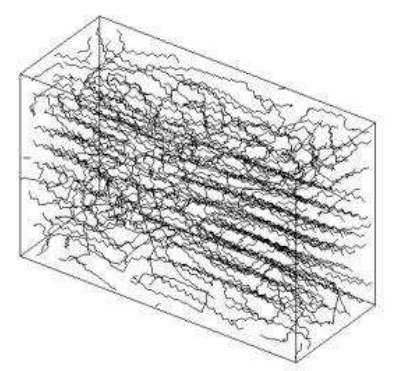

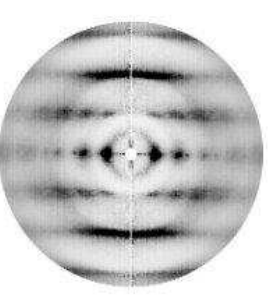

FIG. 3: Snapshots (left), and the structure functions (right) averaged around the draw axis, $S_{2 \mathrm{D}}\left(q_{||}, q_{\perp}\right)$. 
density at the position $\mathbf{r}, \rho(\mathbf{r})$, is defined by the relation $\rho(\mathbf{r})=\sum_{j=1}^{N} \delta\left(\mathbf{r}-\mathbf{r}_{j}\right)$, where $\mathbf{r}_{j}$ represents the position of the $j$-th atom. Here we consider the structure function averaged around the draw axis, $S_{2 \mathrm{D}}\left(q_{\|}, q_{\perp}\right)$, where $q_{\|}$and $q_{\perp}$ are the components of the wave vector along the draw axis and perpendicular to it, respectively. The intensity profile at 10 ps (Fig. 3 (a)) indicates that the system is initially in the oriented amorphous state. We readily notice reflections on the equator at $q_{\perp}=14 \mathrm{~nm}^{-1}$ which are attributed to the nearest neighbor interchain length, and those on the meridian at $q_{\|}=52 \mathrm{~nm}^{-1}$ which correspond to half the fiber period of the polymethylene chain. At 4 ns, Fig. 3(b), broad layer streaks are visible along the layer line at $q_{\|}=26 \mathrm{~nm}^{-1}$, and the reflections at $q_{\perp}=29 \mathrm{~nm}^{-1}$ on the equator are slightly sharper and stronger than in Fig. 3(a). It is clearly found in the snapshots that the degree of the order at 4 ns is considerably higher than that at 10 ps. At 8 ns, Fig. 3 (c), the layer streaks at $q_{\|}=26 \mathrm{~nm}^{-1}$ become much more evident, and the equatorial reflections around $q_{\perp}=29 \mathrm{~nm}^{-1}$ come to sprit. Such diffraction pattern is quite similar to that observed in the hexagonal phase of PE, which appears under high pressure and temperature [24] or under high extension 25]. The snapshot in Fig. 3 (c) shows that the nearby chains tend to orient along the draw axis and form crystalline domains, which emerged around 4 ns and gradually became lager. After 10 ns, marked intensity contrast appears on the streak at $q_{\|}=26 \mathrm{~nm}^{-1}$, and finally at $30 \mathrm{~ns}$ the diffraction come to show ordered crystalline pattern (Fig. $3(\mathrm{~d})$ ) showing the development of high longitudinal order in the crystal.

Around 15 ns the well-developed crystalline layer nearly filled the system. With further development of order, the amount of amorphous segments decreases. But, due to the periodic boundary condition, several parts of the layer surface contacted with the opposite layer surface of itself, which prevent the shrinkage of the MD cell along the draw direction and gives rise to the density decrease in the amorphous phase (Figs. 2 (b), 3 (d)).

In summary, we have performed the MD simulations of the polymer crystallization from the oriented amorphous states. We have succeeded, for the first time, in reproducing the fiber structure of stacked lamellae with ordered crystalline state. We have observed that, after initial relaxation for about 4 ns, the hexagonal order first appears and grows until around $10 \mathrm{~ns}$, and then develops the highly crystalline state through longitudinal adjustments of the parallel chains in the crystal. Analysis of the fiber structure obtained, such as the conformation of tie chains and the structure of the interlamellar amorphous phase, and the detailed molecular process of fiber formation are also very important problems, and they will be published in a separated paper.

This work was supported by a Grant-in-Aid for Science Research on Priority Areas, "Mechanism of Polymer Crystallization" (Grants Nos. 12127203, 12127204 and 12127206) from The Ministry of Education, Science, Sports and Culture of Japan.
[1] D.Rigby and R.J.Roe, J.Chem.Phys. 89, 5280 (1988)

[2] K. Esselink, P. A. J. Hilbers, and B. W. H. van Beest, J. Chem. Phys. 101, 9033 (1994)

[3] H. Takeuchi, J. Chem. Phys. 109, 5614 (1998)

[4] T. Shimizu and T. Yamamoto, J. Chem. Phys. 113, 3351 (2000)

[5] H. Z. Li and T. Yamamoto, J. Chem. Phys. 114, 5774 (2001)

[6] N. Waheed, M. S. Lavine, and G. C. Rutledge, J. Chem. Phys.(to be published)

[7] T. A. Kavassalis and P. R. Sundararajan, Macromolecules 26, 4144 (1993)

[8] C. Liu and M. Muthukumar, J. Chem. Phys. 109, 2536 (1998)

[9] P. Welch and M. Muthukumar, Phys. Rev. Lett. 87, 218302 (2001)

[10] T. Yamamoto, J. Chem. Phys. 107, 2653 (1997); ibid. 109, 4638 (1998)

[11] J. P. K. Doye and D. Frenkel, J. Chem. Phys. 110, 2692(1999); ibid. 110, 7073(1999).

[12] H. Meyer and F. Muller-Plathe, J. Chem. Phys. 115, 7807 (2001)

[13] T. Yamamoto, J. Chem. Phys. 115, 8675 (2001)
[14] A. Koyama, T. Yamamoto, K. Fukao, and Y. Miyamoto, J. Chem. Phys. 115, 560 (2001)

[15] M. Imai, K. Mori, T. Mizukami, K. Kaji and T. Kanaya, Polymer 33, 4451 (1992)

[16] K. Fukao and Y. Miyamoto, Phys. Rev. Lett. 79, 4613 (1997)

[17] K. Tashiro, S. Sasaki, N. Gose, and M. Kobayashi, Polymer Journal (Tokyo) 30, 485 (1998)

[18] P. D. Olmsted et. al. Phys. Rev. Lett. 81, 373 (1998)

[19] A. Mahendrasingam et. al. Polymer 41, 7803 (2000)

[20] S. Gautam, S. Baljepalli, and G. C. Rutledge, Macromolecules 33, 9136 (2000)

[21] H. J. C. Berendsen, J. P. M. Postma, W. F. van Gunsteren, A. Dinola, and J. R. Haak, J. Chem. Phys. 81, 3684 (1984)

[22] D. N. Theodorou and U. W. Suter, Macromolecules 18, 1467 (1985)

[23] I.Ogura and T.Yamamoto, Polymer, 36, 1375 (1995)

[24] T.Yamamoto, J. Macromol. Sci. Phys. B13, 487(1979)

[25] J. Pennings and A. Zwijnenburg, J. Polym. Sci, Polym. Phys. Ed. 17, 1011 (1979) 